\title{
Río Santiago, un paisaje fluvial en colapso como oportunidad de recuperación urbana
}

\author{
Rio Santiago, a river landscape in collapse as an \\ opportunity for urban recovery
}

Resumen

Autores:

Isamar Herrera Piñuelas** iherrera@esarq.edu.mx

Alfred Esteller Agustí*** esteller@iteso.mx

Adolfo Vigil de Insausti* advide@urb.upv.es

* Universitat Politècnica de Valencia **ESARQ, Escuela Superior de Arquitectura ***ITESO, Universidad Jesuita de Guadalajara

*España ***éxico **** México

Recibido: 14/Mar/2021 Aceptado: 29/Jun/2021
M éxico, territorio que goza de extensión y diversidad, es escenario de múltiples bellezas naturales asociadas a cuerpos de agua cuya magnitud los convierten en atractivos naturales. En contraparte, hoy en día algunos de estos cuerpos de agua presentan tal deterioro ambiental que resultan ser no solo un impacto negativo para el ecosistema natural sino de alto riesgo para el contexto urbano inmediato. El río Santiago y sus expresiones fluviales, después de ser considerado como el Niágara Mexicano, se ha convertido en las últimas décadas en un lugar irrespirable, tras recibir los residuos industriales y urbanos de la zona. Esta condición ha sido determinante para las poblaciones, las cuales adolecen de la problemática de la contaminación con graves efectos en la salud. El estudio presenta una serie de posibilidades para la recuperación del paisaje fluvial como una estrategia para la mejora de la sanidad ambiental utilizando como herramienta de análisis la perspectiva urbana.

Palabras clave: urbanismo; paisaje; ecología urbana; cuerpos de agua; sanidad ambiental.

\section{Abstract:}

Mexico, an extensive and diverse land, is the scene of multiple natural beauties linked to waterscapes whose magnitude make them natural attractions. But, today some of these water bodies presents such environmental deterioration turning them into not only a negative impact for the natural ecosystem but also a high risk for the immediate urban context. Santiago river and its fluvial expressions, after being considered the Mexican Niagara, in recent decades has become an unbreathable place, after receiving industrial and urban waste from the area. This condition has been decisive for the populations, which suffer the pollution problem with serious health effects. The study presents a series of possibilities for the recovery of the waterscape as a strategy for improving environmental health using the urban perspective as an analysis's tool.

Keywords: urban planning; landscape; urban ecology; waterbody; environmental health. 


\section{Introducción}

La Sierra Madre Occidental Mexicana acoge uno de los cuerpos de agua más importantes de México por su capacidad de afluente, la cuenca Lerma. Con una superficie de $5,354 \mathrm{~km}^{2}$, es abastecedor de agua potable a 33 municipios que representan alrededor de 2,7 millones de habitantes, e incorpora una de las masas forestales más importantes del país, albergando aproximadamente $2 \%$ de la biodiversidad mundial, considerando además que $10 \%$ de ellas son de carácter endémico (Comisión de la Cuenca del Río Lerma, 2020). El río Lerma termina su recorrido en el Lago de Chapala, ubicado en Jalisco, México. A partir de este punto comienza su recorrido el río Santiago. Estos tres cuerpos de agua, desde 1938 son propiedad nacional, declarado por el Diario Oficial de la Federación de México. La cuenca en su totalidad representa una presión por los recursos naturales, pues su uso está compartido entre los usuarios de seis estados: Guanajuato, Michoacán, Jalisco, Estado de México, Querétaro y Nayarit, generando solo en el primer tramo, Lerma - Chapala, el $47 \%$ del Valor Agregado Censal Bruto nacional (Cotler et al., 2006).

Sin embargo, los recursos hidráulicos de la cuenca han sufrido deterioro en su calidad a causa de descargas residuales. Según datos oficiales de la administración pública, se estima la responsabilidad al aporte de aproximadamente 741 empresas del área, que en su mayoría no cumplen con el marco legal y normativo. Se trata de industrias de tipo metálica, electrónica, química, plásticos, muebleras y alimentos, entre otros (McCulligh, 2017), además de los aportes provenientes de la industria agrícola, así como de asentamientos humanos. La situación representa un problema sistémico, pues la representatividad económica que tiene la zona por su desempeño industrial presenta una situación insostenible para las poblaciones alrededor del río. El impacto hacia los ciudadanos es un problema persistente, el cual, además, con el pasar del tiempo se convierte en una situación que agrava e intensifica el malestar colectivo de los pobladores. La salud, como derecho humano fundamental, es una de las principales demandas de las comunidades, pues la presencia de continuas enfermedades incrementa la preocupación y genera un estado continuo de incertidumbre. Esto, a su vez, ha provocado que la situación se posicione actualmente en la mirada de diversos organismos públicos y privados, así como también capturar la atención de los medios de comunicación, nacionales e internacionales.

Si bien, a raíz de potentes investigaciones sobre la calidad del agua y su relación con la salud humana, se han desprendido reflexiones desde la óptica legislativa, sería también importante considerar aquellos aportes en donde se expongan posibilidades desde un enfoque de entendimiento del territorio y de su relación con las dinámicas urbanas.

\section{Métodos}

Debido a la cantidad de información producto de investigaciones del área, se realiza una investigación documental mediante artículos de divulgación científica sobre las condiciones del río, considerando únicamente factores ambientales de tipo químico y biológico relacionados con la calidad del agua del tramo SantiagoChapala. De forma adicional, se toman en consideración aquellas investigaciones que relacionen estos factores hacia las afectaciones a la salud humana.

En consecuencia, respecto al impacto social que ha tenido la problemática, la investigación se complementa mediante la revisión documental generada debido a la fuerza mediática de la situación, y se realiza el análisis de material de prensa, considerando un proceso de selección y de discriminación de la información en función del posicionamiento y de la fiabilidad histórica de las fuentes.

Una vez contextualizada la problemática a través de la búsqueda documental, se realiza un estudio de las zonas urbanas vinculadas al río Santiago, tomando en consideración los 12 municipios determinados como prioritarios por el programa constituido por el gobierno del estado de Jalisco Revive el Río Santiago: Zapotlanejo, Zapopan, Guadalajara, Tonalá, Tlaquepaque, Tlajomulco, El Salto, Juanacatlán, Ixtlahuacán, Chapala, Zapotlán del Rey y Poncitlán; analizando las comunidades ubicadas a menos de 150 metros del margen del río y con una altitud inferior a 20 metros de diferencia (Figura 1 y 2).

La recopilación de información sobre la calidad del agua se realizó mediante tres plataformas de información estadística de México: SIG metro del Instituto Metropolitano de Planeación del Área Metropolitana de Guadalajara, el Atlas Nacional de Riesgo de la Secretaría de Seguridad y Protección Ciudadana, y el mapa digital de México del Instituto Nacional de Estadística, Geografía e Informática. A través de ellos se recuperó la información relativa a los análisis realizados a lo largo del río durante el periodo 2000-2020. Esta información fue contrastada posteriormente con el análisis de crecimiento superficial urbano, cuyos datos fueron recuperados a partir de los datos demográficos-históricos del Instituto Nacional de Estadística, Geografía e Informática en conjunto con la cartografía y ortofotos satelitales incluidas en SIG metro, el Atlas Nacional de Riesgo y el mapa digital de México. A partir de esta información se maquetan tres gráficos compilatorios como parte del diagnóstico territorial, tomando en consideración la contrastación de información relacionada al estado de la calidad del agua y sus efectos a las dinámicas socio-urbanas de la zona. 


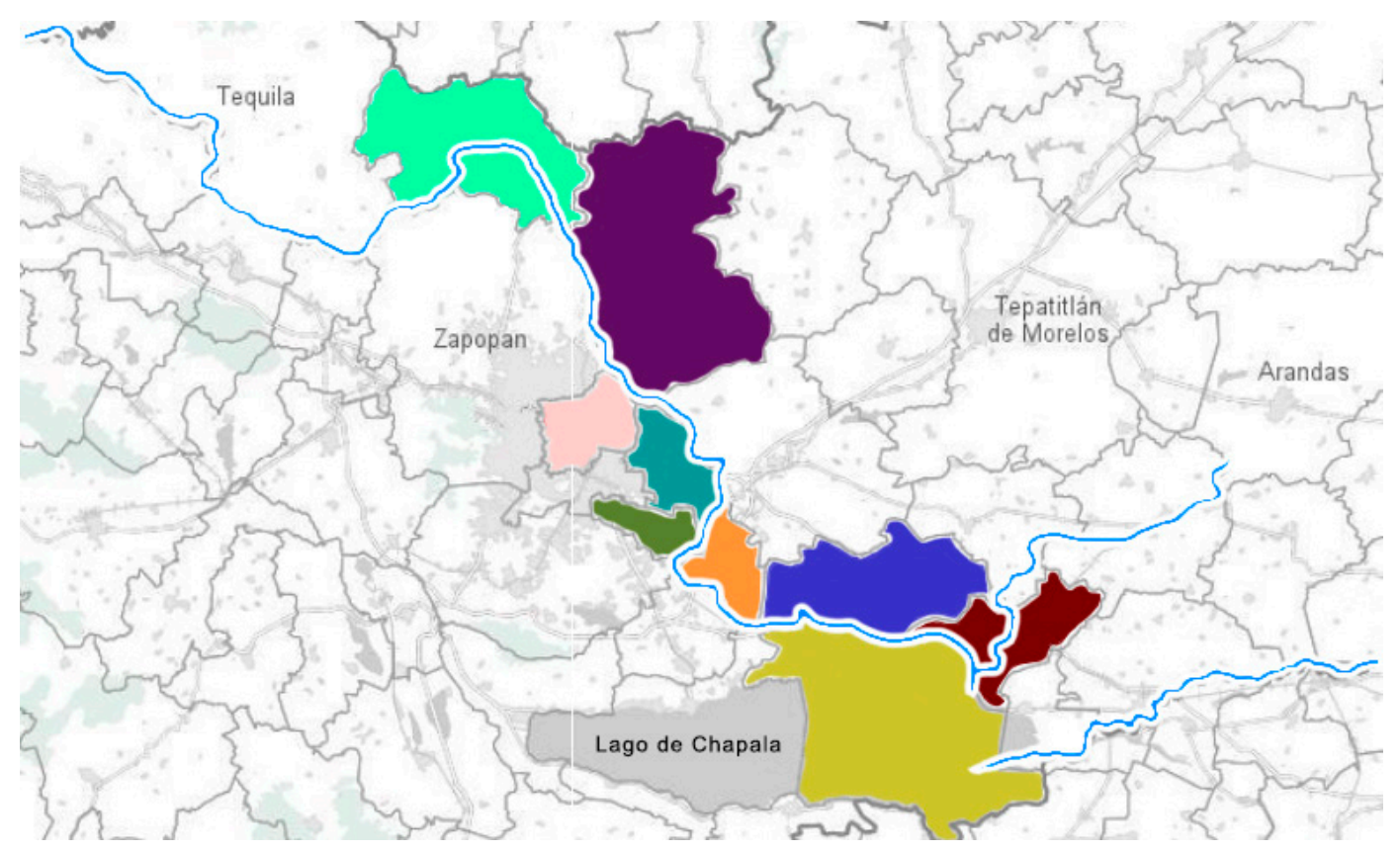

Figura 1: Municipios de las comunidades estudiadas

Fuente: Autoría propia (2021)

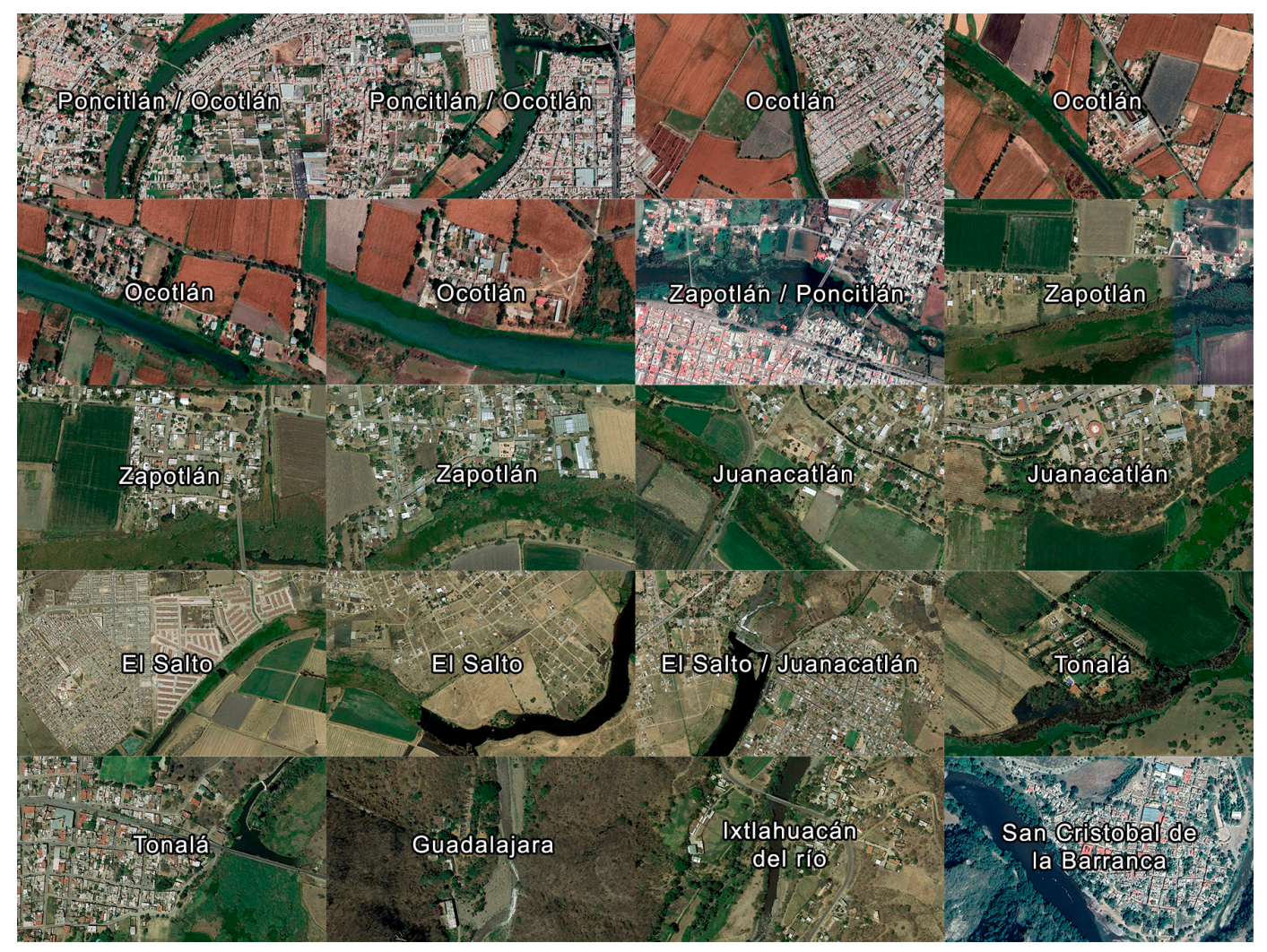

Figura 2: Sectores estudiados

Fuente: Autoría propia con ortofotos de Google 


\section{Resultados}

\subsection{Acercamiento a la distopía continuada del río Lerma}

En 2011, el Gobierno del Estado de México en conjunto con la imagen identitaria del Estado de México, EDOMEX y la Secretaria de Obra Pública, crean la Comisión Coordinadora para la Recuperación Ecológica de la Cuenca hidrológica del río Lerma como parte del acuerdo del Gobierno del Estado de México del 18 de agosto de 1990, con el objeto de contribuir al saneamiento de la Cuenca correspondiente al Estado de México. En esta comisión, además de encontrarse directrices y planes de acción, se encuentran publicaciones e investigaciones en torno al río Lerma, algunas de ellas, como el Atlas de la Cuenca del río Lerma en el Estado de México (compendio) y los 7 tomos complementarios sobre la situación urbano-social, son utilizados para relatar la situación geográfica y las consecuencias del estado de río Lerma. El Gobierno del Estado de México (2011) describe la ubicación y trayectoria del río dando inicio en la laguna de Chiganhuapan, en el contexto de la sierra de Santa Ana Nichi, conectando con el Nevado de Toluca y los volcanes San Antonio y La Calera en el costado suroeste. Siguiendo el cauce del río hacia el norte está la laguna de San Bartolo, hasta donde se cree que llegaba la extensión lacustre. La cuenca de este sistema-130 mi kilómetros cuadrados- abarca porciones de los estados de México, Michoacán, Querétaro, Guanajuato, Jalisco, Aguascalientes, Zacatecas, Durango y Nayarit, entidades que reciben el benéfico paso del agua por su territorio o sus cercanías, y a la vez contribuyen con su aportación al caudal del Lerma.
El río Lerma alimenta de agua a aproximadamente 3 millones de habitantes; por tanto, es precursor de una de las problemáticas de salud pública más delicadas del Estado de México, según diversas notas de prensa respaldadas por investigaciones de carácter toxicológico. Laura Velásquez expone a través del diario El Heraldo el 14 de abril del 2019, que el río Lerma ha pasado de ser soporte vital a totalmente lo opuesto debido a que los núcleos urbanos vierten en él sus aguas residuales, $60 \%$ de ellas sin tratar, convirtiéndose la contaminación de tipo orgánica en el principal contaminante del cuerpo de agua. Un reporte realizado por estudiantes de la Universidad Nacional de México y la Universidad Autónoma Metropolitana, analizando concentración de oxígeno, conductividad, cantidad de biomasa, concentración de nitrógeno con amonio, nitrógeno como nitratos y fósforo como ortofosfato, y en relación a las zonas y temporalidad del muestreo, concluyó que el agua del río Lerma está altamente impactada por desechos industriales, urbanos y agrícolas. Acorde con la escala de clasificación de la Secretaría de Medio Ambiente y Recursos Naturales sobre la calidad del agua para usos específicos, el agua del río Lerma presentó aguas de altamente contaminadas a contaminadas, lo que coincide con la evaluación del Índice Biótico Extendido (IBE), que las clasifica como aguas con alta y severa contaminación (López-Hernández et al., 2006). El río Santiago pertenece, según la Comisión Nacional de Agua, a la Región hidrológica-Administrativa VIII Lerma Santiago Pacífico (Figura 3). Como todo sistema fluvial, la cuenca hidrológica Lerma-Santiago-Pacífico está compuesta por diversos vasos conectores que se encargan del balance hídrico y de cumplir con su función transportadora de energía, agua y sedimentos, lo que genera en el caso del río Lerma una prolongación de la problemática que se

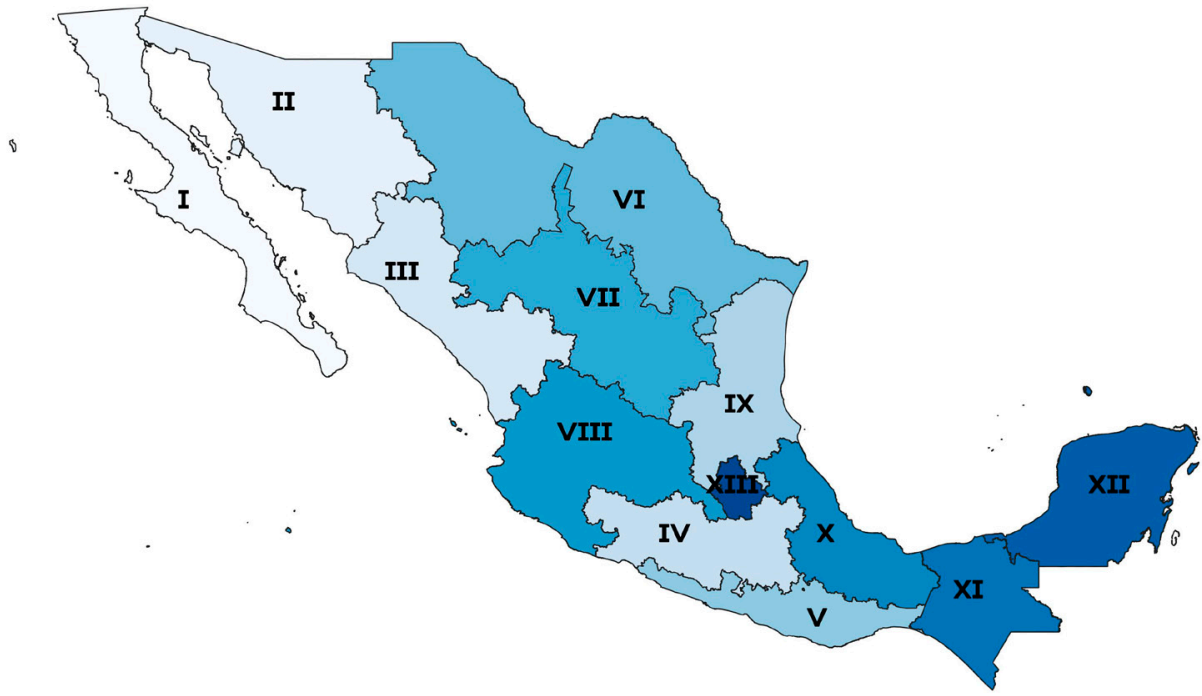

Figura 3: Regiones hidrológicas-administrativas

Fuente: Comisión Estatal del Agua Jalisco. https://www.ceajalisco.gob.mx/contenido/cuencas jalisco/ (2005) 
transfiere río abajo al resto de la cuenca hidrológica. De este modo, el Lago de Chapala es un vaso conector del río Lerma con el río Santiago (Figura 4).

Una vez que el río Lerma se deposita en el Lago de Chapala, el vertido de contaminantes al río Santiago continúa, pues se suman los aportes fluviales que tiene el río Zula, el cual es otro caso que presenta fuertes impactos por descargas municipales procedentes, principalmente, de fábricas de tequila y de industrias porcinas y avícolas, situación ocasionada por el déficit de plantas y de sistemas de tratamiento de los desechos (Guzmán-Arroyo y Peniche-Camps, 2009).

La suma de estas condiciones desafortunadas, de origen fundamentalmente antropogénico, supone para el río Santiago una acumulación de aportes tóxicos al afluente, un escenario que supone una magnificación de la problemática debido a las necesidades de las poblaciones, las cuales requieren de esa agua como parte importante para el desarrollo de sus actividades. Esto genera una especie de relación similar al parasitismo, lo que produce incontables problemas a la salud ambienta y humana, provocando que los paisajes fluviales sean percibidos como víctimas y victimarios de la relación con los centros urbanos.

En México, la descarga a los ríos de aguas residuales -tanto residenciales como industriales- sin análisis ni tratamiento previo, es un problema histórico. Esta situación se agrava de bido a la permisividad gubernamental a nivel federal, estatal y municipal, así como a la falta de cumplimiento de la de por sí laxa normatividad en materia de agua, ya que las normas de descarga -NOM001 y NOM002- regulan de acuerdo con pocos criterios de contaminación, como la Demanda Química de Oxígeno (DQO) y la demanda bioquímica de Oxígeno (DBO), criterios internacionales que no identifican sustancias específicas en el agua (Guevara et al., 2014, p.3)

Por lo que no se consideran, por ejemplo, Coliformes Fecales (CF) (Tabla 1) ni Sólidos Suspendidos Totales (SST).

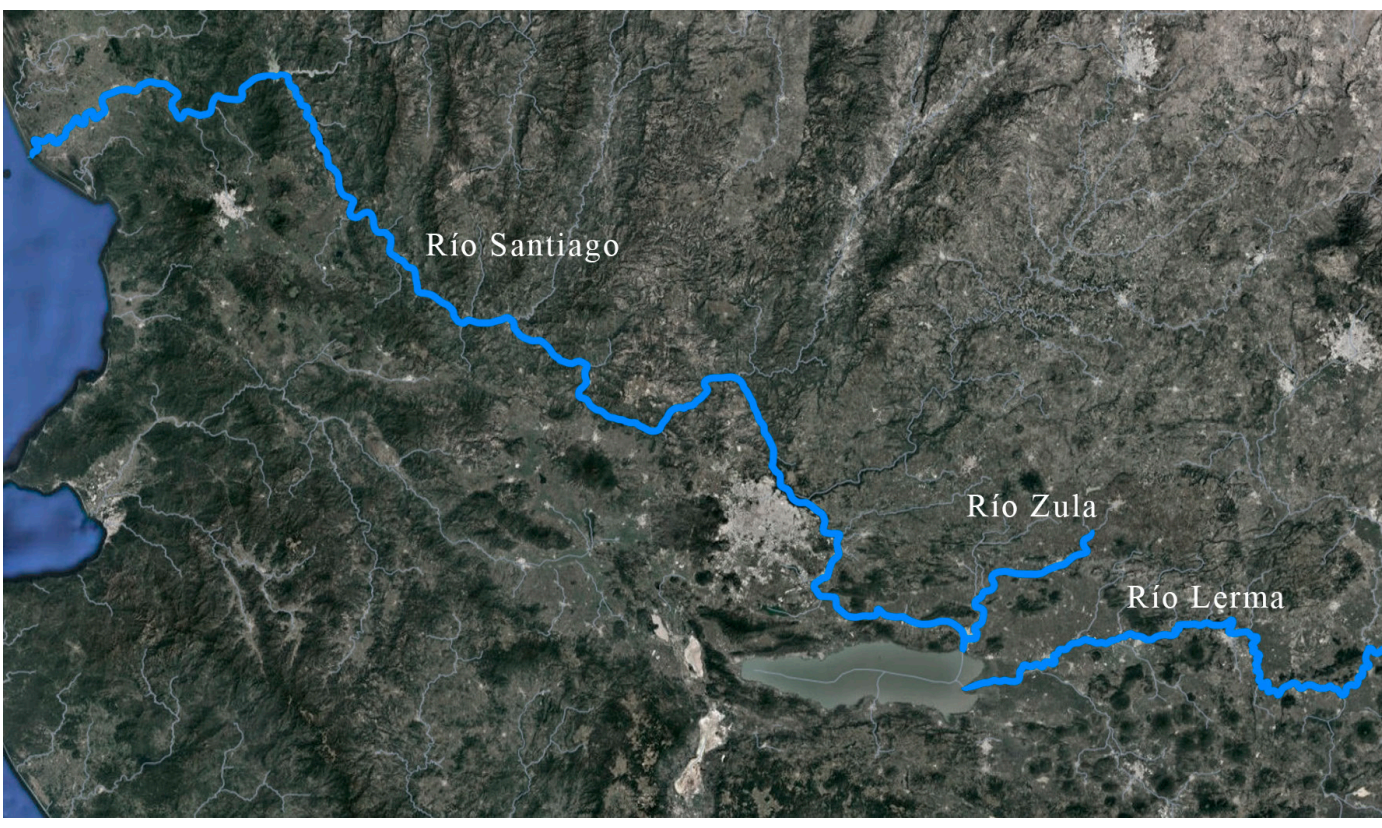

Por otra parte, varios estudios sobre la calidad del agua del lago de Chapala indican su estado y el riesgo que supone a la sanidad ambiental. Un estudio de Quiroz-Castelán et al. (2004) revela que la concentración elevada de grasas, aceites y cloruros son indicadores de contaminación por aguas residuales, y que desde hace tiempo el Lago de Chapala ha mostrado valores continuos que evidencian fase de eutrofia como resultado del continuo aporte de contaminantes o nutrientes, pues si bien pueden ser de origen doméstico o industrial, también la agricultura representa un aporte a la eutrofización.

Los estudios que arrojan este tipo de resultados donde se muestra el estado de eutrofia de los cuerpos lacustres, exponen una situación que se retroalimenta como consecuencia de los aportes industriales, agrícolas y residuales de los asentamientos urbanos. Es un círculo vicioso en donde los aportes de materia orgánica incrementa la cantidad de nutrientes, y por tanto aceleran la reproducción de los productores primarios, quienes a su vez aumentan la demanda de oxígeno, lo que genera consecuentemente, en el caso del Lago de Chapala, una sobrepoblación de lirio, formando coberturas vegetales sobre el lago que reducen la cantidad de luz a las regiones profundas del mismo; luz necesaria para los organismos fotosintéticos, provocando su muerte, generando durante años un ecosistema altamente eutrofizado, con bajos niveles de oxígeno y altos niveles de fósforo y de nitrógeno. Según un estudio realizado por de Anda y Maniak (2007), a través de un método de evaluación de las aguas se demuestra que el lago ha perdido su capacidad de remover el fósforo total. Igualmente se determinó que la cantidad promedio de fosfato en el lago representa aproximadamente un 70\% del fósforo total, lo cual supone que el lago mantiene altos niveles de disponibilidad biológica en la columna de agua.

Figura 4: Ubicación del Río Santiago

Fuente: Autoría propia sobre cartografía del Instituto Nacional de Estadística y Geografía (2020) 


\begin{tabular}{|c|c|c|c|c|c|}
\hline Región hidrológico-administrativa & Excelente & Buena Calidad & Aceptable & Contaminada & $\begin{array}{l}\text { Fuertemente } \\
\text { contaminada }\end{array}$ \\
\hline I Península de Baja California & 67,3 & 3,1 & 10,6 & 11,5 & 7,5 \\
\hline II Noroeste & 32,7 & 7,2 & 13,1 & 26,1 & 20,9 \\
\hline III Pacífico Norte & 13,0 & 6,0 & 19,9 & 44,9 & 16,2 \\
\hline IV Balsas & 8,4 & 2,0 & 7,8 & 19,2 & 62,6 \\
\hline V Pacífico Sur & 43,9 & 3,8 & 9,0 & 26,7 & 16,6 \\
\hline VI Río Bravo & 25,6 & 6,9 & 23,5 & 27,4 & 16,6 \\
\hline VII Cuencas Centrales del Norte & 18,1 & 5,5 & 30,9 & 36,4 & 9,1 \\
\hline VIII Lerma Santiago Pacífico & 12,5 & 5,3 & 10,6 & 26,2 & 45,4 \\
\hline IX Golfo Norte & 21,3 & 4,5 & 17,2 & 29,6 & 27,4 \\
\hline x Golfo Centro & 11,6 & 2,7 & 11,0 & 37,5 & 37,2 \\
\hline XI Frontera Sur & 29,3 & 6,0 & 24,5 & 29,9 & 10,3 \\
\hline XII Penínsual de Yucatán & 57,1 & 11,5 & 21,5 & 9,4 &, 5 \\
\hline XIII Aguas del Valle de México & 8,5 & 0 & 8,5 & 12,6 & 70,4 \\
\hline
\end{tabular}

Tabla 1: Resultados de CF en las diversas regiones hidrológico-administrativas en México

Fuente: Autoría propia con datos estadísticos de la Comisión Nacional del Agua, México 2018. http://sina.conagua. gob.mx/publicaciones/EAM_2018.pdf (2018)

Por tanto, la situación en la que se encuentra el río Lerma, el río Zula y el lago de Chapala se transporta a las condiciones del cuerpo de agua consiguientes; lo que significa para el río Santiago un transporte de condiciones de insanidad ambiental desde la cuenca hidrológica, además de las condiciones que padece en sí mismo el río Santiago como consecuencia de su ubicación y trayectoria.

El problema del río Santiago ha escalado en magnitud al pasar de los años, convirtiéndose no solo en un tema de salud pública sino trascendiendo a diversos niveles, políticos, económicos y sociales, que se han transformado en otros intereses, de poder, información y criminalidad, debido mayormente a causa de regulaciones que omiten la medición de las descargas industriales, y datos básicos y fundamentales, como podría ser la demanda química y bioquímica de oxígeno. La norma oficial mexicana, NOM001-SEMARNAT-1996, que establece los límites máximos permisibles de contaminantes en las descargas de aguas residuales en aguas y bienes nacionales, tiene deficiencias en los límites y componentes regulados, pues se limita el margen de protección del medio ambiente, hasta el punto de que tolueno, benceno y ftalatos son vertidos sin regulación alguna pese a su reconocido daño a la salud humana (McCulligh, 2017); así como el arsénico, presente en la composición del suelo de Jalisco debido a la zona volcánica a la que pertenece, y con lo que, aunque no afecte de forma directa al agua potable, se especula con la relación de la recarga de mantos freáticos de estas concentraciones asociadas al río.

La región Lerma-Santiago-Pacífico acumula malas gestiones y pocas oportunidades de mejora, con un río que atormenta la fauna silvestre y asentamientos humanos. Esta condición ha generado lo que se conoce como desacoplamiento entre la sociedad urbana $y$ la naturaleza, una relación disociativa y con daños bilaterales.

\subsection{Cacotopía a la orilla de un río}

La historia de las condiciones actuales del río Santiago es una sumatoria del abandono político, de los muchos huecos legislativos y de un cúmulo de necesidades urbanas asociadas a los núcleos poblacionales aledaños al río. Según los estudios de una investigadora que ha dedicado gran parte de su vida al estudio del río Santiago, McCulligh (2007), se describe que el río Santiago fue base fundamental para el asentamiento de varias poblaciones debido a que durante el siglo XIX, tras la instalación una hidroeléctrica, la más importante del país y la primera de América Latina, se generó una importante atracción de inversionistas que produjeron la conformación del corredor industrial, todo aquello con la ilusión de que la calidad de vida en la zona mejoraría. La importancia para los asentamientos poblaciones, según la Comisión Nacional de Agua (2012), seguirá persistiendo pues se calcula que para 2030 el $70 \%$ del crecimiento poblacional ocurrirá en las regiones hidrológico-administrativas de Lerma-Santiago-Pacífico, Aguas del Valle de México, Río Bravo y Península de Baja California.

Esta situación a lo largo del tiempo merma no solo en la salud sino en la integridad humana de las poblaciones afectadas. El conflicto del agua refleja una situación compleja de tipo urbano, industrial, agroindustrial y hasta turístico. Este tipo de decisiones en el entorno urbano representan zonas de sacrificio a costa de la salud ecosistémica. Este término-zonas de sacrificio- ha sido utilizado a manera de concepto que justifica, a nivel social y ambiental, aquellas áreas urbanas en el abandono y a la merced de la fuente contaminante que generalmente es la industria, en donde los habitantes de la zona son quienes corren un riesgo extremo por contacto (McCulligh, 2017). La relación antropogénica que se ha formulado en la zona del río Santiago ha terminado por lesionar las dos partes de la ribera, y como círculo vicioso no consigue detener las afecciones ocasionadas 
en ninguna de las dos vías: ni la de la vida acuática ni la humana. La misma investigadora y especialista, McCulligh (2013), señala que en el caso de México es evidente cómo en repetidas ocasiones los actores gubernamentales priorizan el crecimiento económico frente a cualquier protección ambiental, generando una sensación de que es imposible conciliar la conservación ambiental con el desarrollo económico del país (McCulligh, 2013). El río Santiago resulta ser uno de los diversos casos en México donde el recurso hídrico genera una constante tensión entre la vida de las ciudades, el crecimiento urbano, el uso y la gestión sostenible del recurso.

Las afecciones que se presentan en el eje urbano y humano son multiescala, comenzando por una violación de derechos humanos, tal como fue presentado a través de una queja a la Comisión Estatal de Derechos Humanos de Jalisco por parte de los pueblos afectados por el río Santiago.

De modo similar a esta queja, una serie de otras llamadas de atención han recurrido a otras vías, incluyendo recientemente a las universidades, las cuales se han convertido en el grupo de presión más importante, y en las que se puede facilitar la exposición del caso mediante nuevos y diversos trabajos de campo, la mayoría de ellos en el orden de la recopilación de datos físicos y químicos de las condiciones del agua; pero por otra parte, y de suma importancia, están aquellos relacionados al bienestar social de la comunidad. Un análisis de campo realizado por Martínez-González y Hernández-González (2009) arrojó algunos resultados en relación con el espacio público: "en cuanto a la dimensión social de las necesidades de estima, constatamos que las actividades de convivencia familiar y comunitaria en torno al río han dejado de practicarse y se han perdido algunos elementos que le daban identidad a esta población" (p.728). En ello se debe resaltar que se transgrede a la vocación del espacio público, en tanto que también se especula sobre las afecciones particulares hacia el interior del espacio privado de las familias, desde el eje emocional y psicológico.

La tensión asociada al futuro, tanto por parte de las comunidades como del corredor industrial, produce especulaciones que generan una sucesión de acciones lentas no exentas de recelo pues, como se puede pensar, la industria vela por los intereses económicos y productivos propios, mientras que los habitantes siguen padeciendo de una situación que paulatinamente se convierte en un cúmulo de vivencias que desembocan en anécdotas terroríficas sobre la tremenda situación de colapso ambiental que les rodea.

El año pasado fuimos al Platanar a los guamúchiles y ya los chiquillos decían mira una vaca muerta, otra vaca muerta, muchísimas vacas muertas (...) Si sales afuera (de la casa), o hueles el río o hueles la basurera, por eso te digo que nos estamos quedando sin vida... Aquí nos llega el olor del río y el de la basurera, nosotros ya estamos inmunizados hasta del olor (Martínez-González y Hernández-González, 2009, pp.723-724).

El problema sobre la seguridad de las poblaciones se vio agudizado a lo largo del 2008, tras la intoxicación por arsénico y causa de muerte de un infante. Sin embargo, el Comité Ciudadano en Defensa Ambiental del Salto declara en prensa que alrededor de 1.500 personas han fallecido desde entonces por padecimientos que se relacionan con la contaminación, presuntamente del río (El informador, 13 de febrero de 2020). De igual forma, la agencia de noticias internacional EFE (3 de marzo de 2020) expresa que en estas comunidades es común escuchar sobre

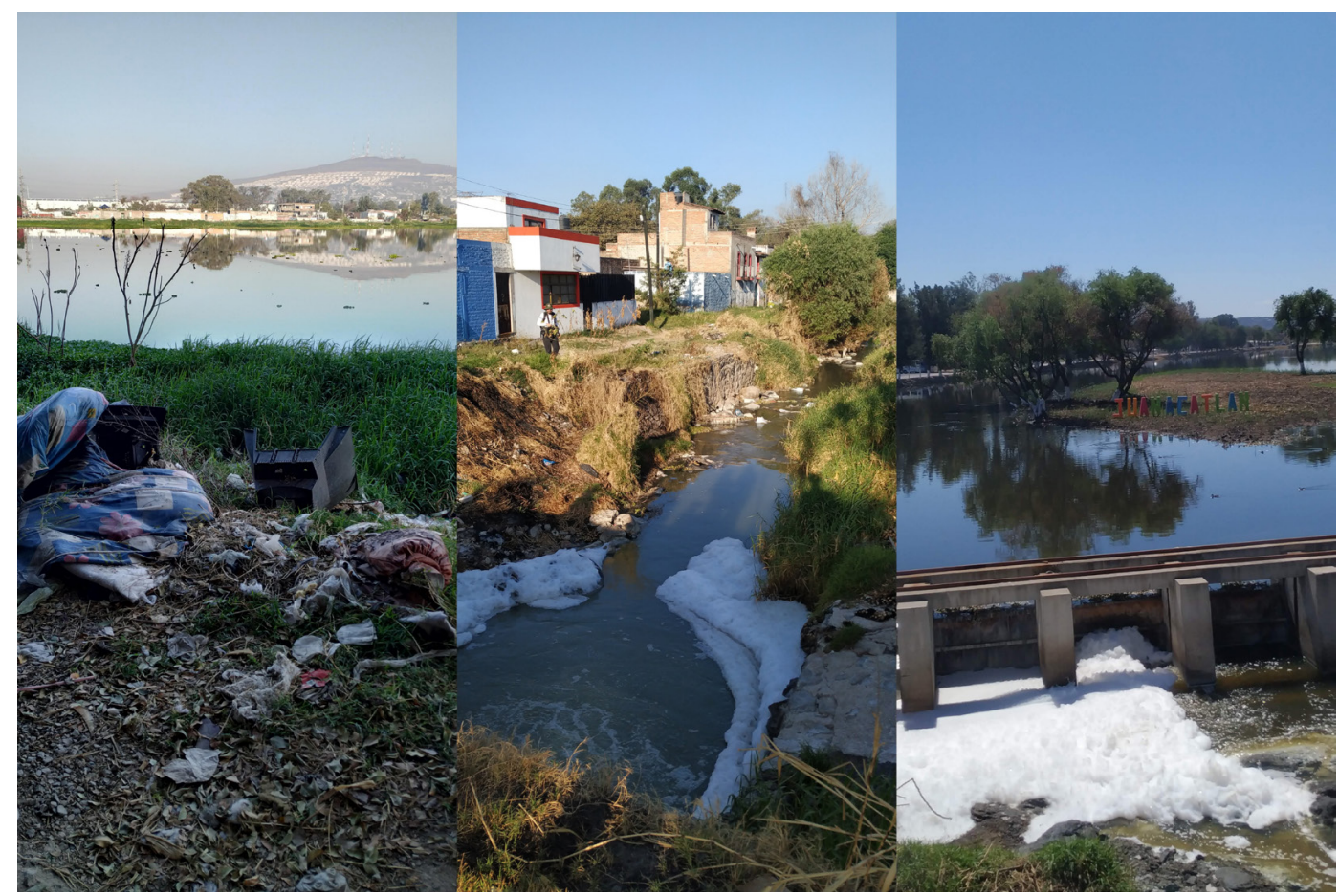

Figura 5: Estado actual del río en varios puntos del recorrido.

Fuente: Jesús Enrique Cueva Lomas(2020) 
algún caso de vecino o familiar quien padece cáncer o alguna enfermedad renal asociada al estado actual de río (Figura 5). En la nota se expone el caso específico del testimonio de una persona diagnosticada de cáncer que sospechan puede ser resultado de la exposición a agua del río Santiago y a las partículas contaminantes suspendidas en el aire.

Un estudio realizado por la Comisión Estatal de Agua de Jalisco en conjunto con la Universidad Autónoma de San Luis Potosí (Domínguez Cortinas, 2010) expone, mediante una serie de indicadores sociales, ambientales y de salud, resultados correspondientes a la población de la zona. En el caso de los infantes, en la dimensión salud los resultados expresan la prevalencia en la mayoría de los casos de enfermedades respiratorias, conjuntivas oculares, alteraciones hematológicas y de habilidades cognitivas, además de presentar en todas las poblaciones analizadas un alto porcentaje de personas con trastornos emocionales y bajo rendimiento escolar. Estos resultados, los relaciona el estudio con determinantes biológicas, donde predominan los patógenos intestinales y la exposición a cadmio, mercurio, plomo y COP (Contaminantes Orgánicos Persistentes), además de algunos otros también presentes en los resultados, y en menor medida: flúor, arsénico, ácido tt-mucónico y ácido hipúrico.

Por otra parte, dentro del mismo estudio los resultados de la dimensión social expresan que las poblaciones presentan ingresos muy bajos, condiciones urbanas insatisfactorias con falta de saneamiento, calles no pavimentadas y hacinamiento; mientras que de la dimensión ambiental destaca una relación entre la presencia de plagas de insectos que, por tanto, genera un uso extensivo de insecticidas domésticos, con énfasis en el combate del mosquito transmisor del dengue, así como otros problemas mayores relacionados a los servicios básicos, como la escasez de agua y la necesidad de quema de basura.

\subsection{Diagnóstico del estado actual}

Como producto del análisis, para el mapeo de los resultados según las muestras de agua analizadas se filtran los datos para mostrar solo aquellos referentes a un estado determinado como contaminado o fuerte contaminado, según los estándares de medición del organismo regulatorio (Figura 6).

Se combinan posteriormente en un gráfico conjunto los datos de evolución anual de los resultados de calidad del agua con la evolución del crecimiento urbano de las poblaciones analizadas (Figura 7).

El Salto y Juanacatlán son los casos muestra de mayor crecimiento urbano en los márgenes del río Santiago (Figura 7), observando la evolución de su mancha urbana en relación a los datos de marginación y porcentaje de vivienda desocupada (Figura 8), queda patente la situación de vivienda abandonada y marginación de los nuevos desarrollos urbanos ubicados en la ribera del río.

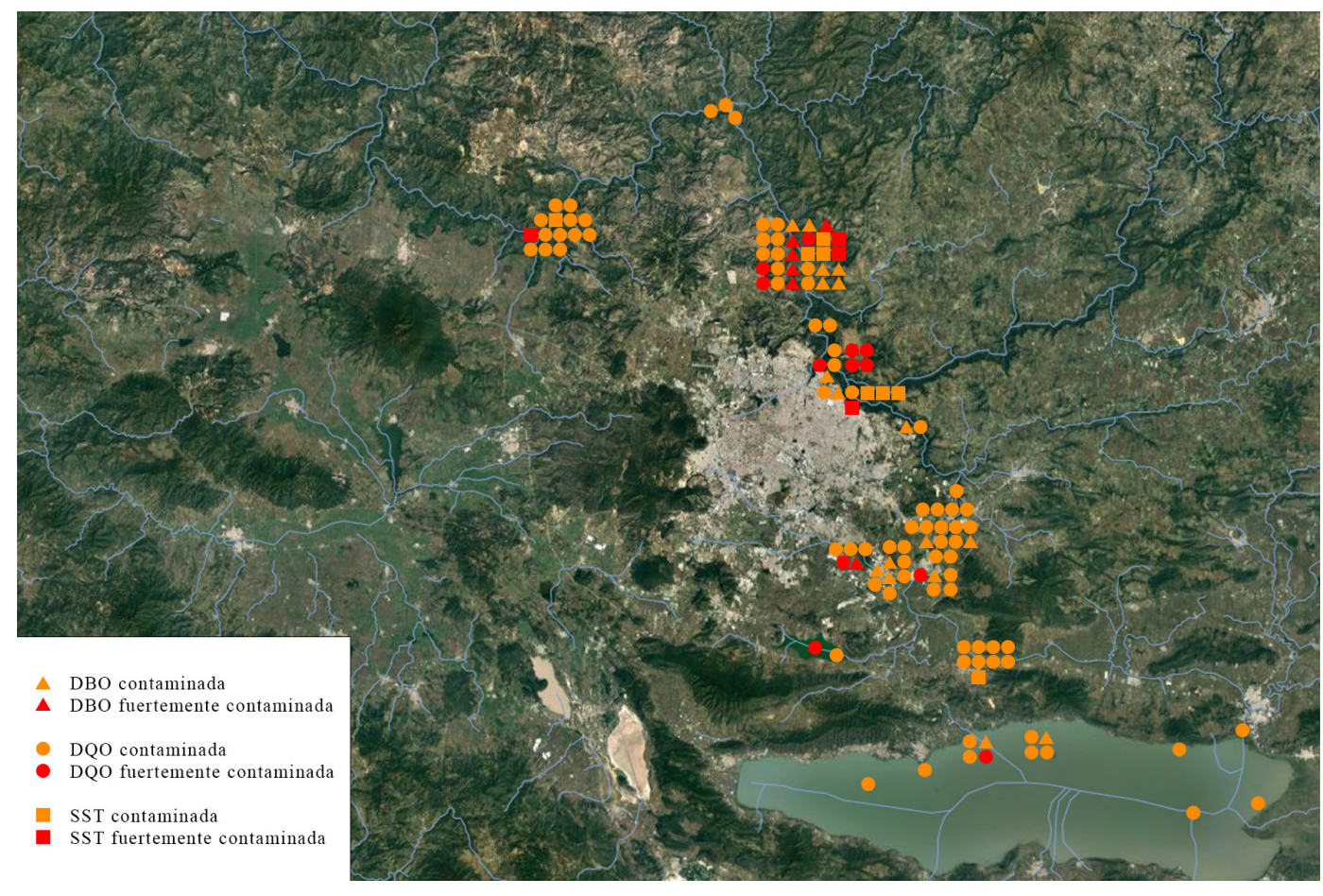

Figura 6: Registros de calidad de agua

Fuente: Autoría propia (2021) 


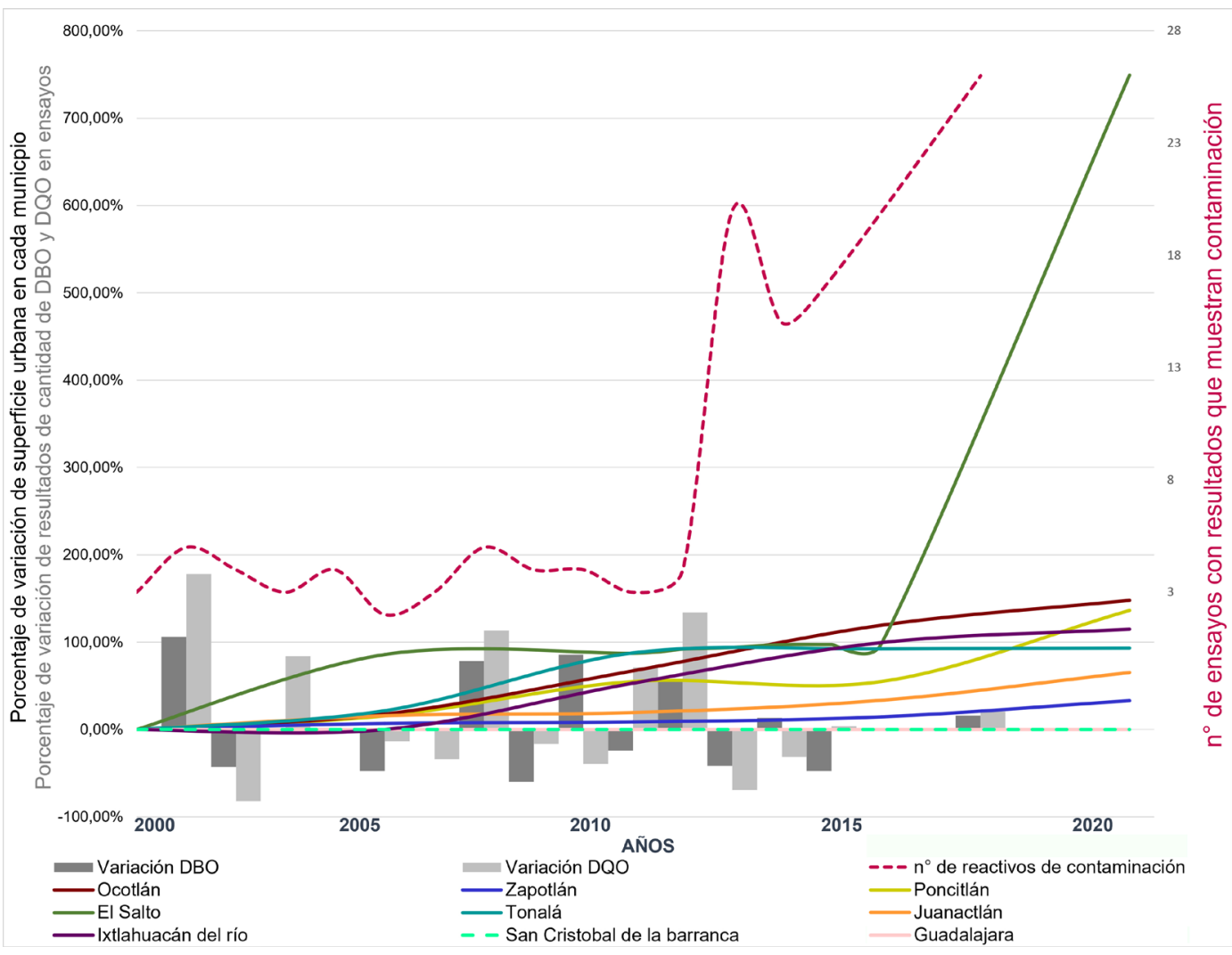

Figura 7: Porcentaje de crecimiento urbano contra calidad de agua según DQO y DBO Fuente: Autoría propia (2021)

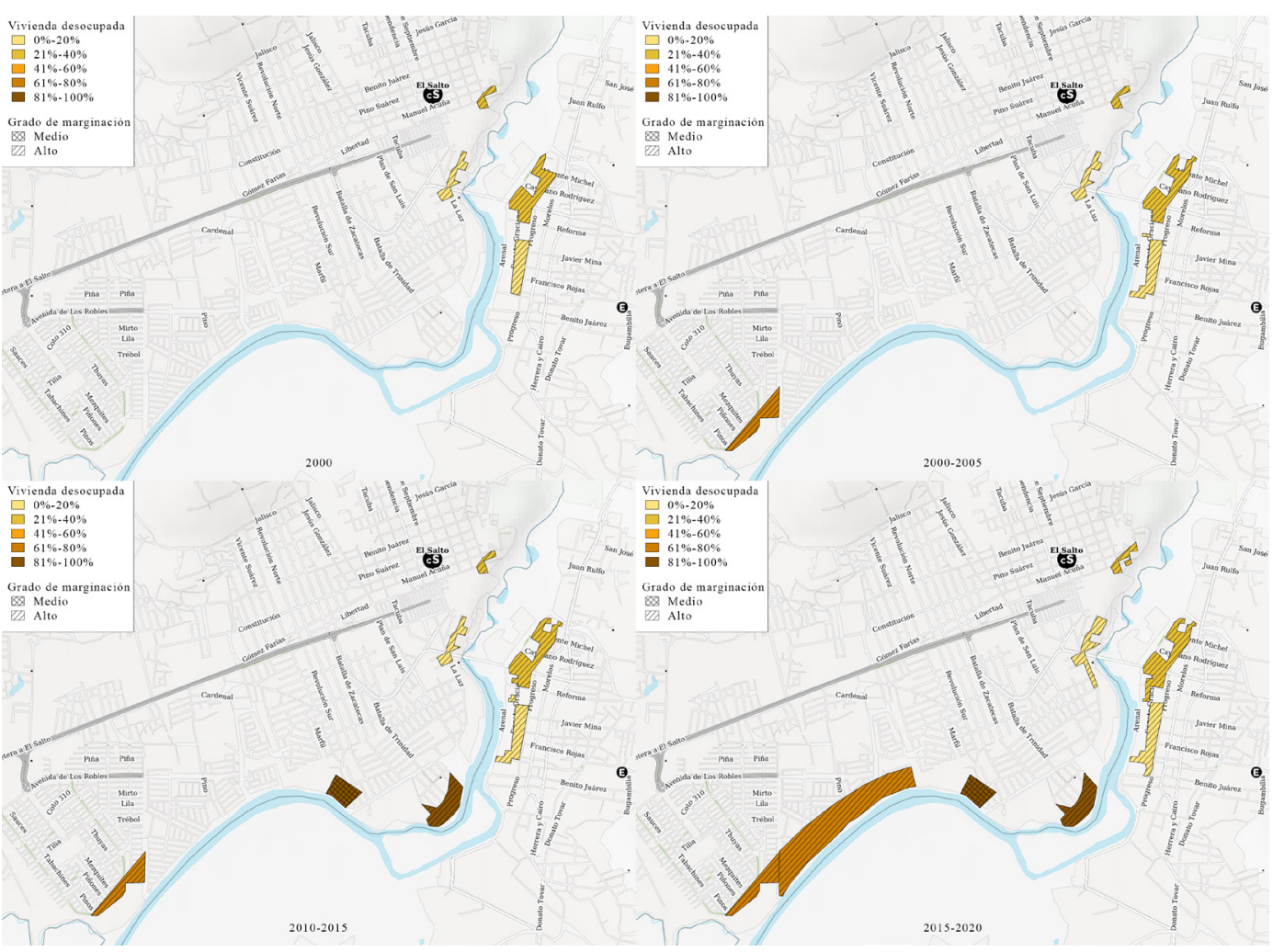

Figura 8: Evolución de mancha urbana, marginación y vivienda desocupada Fuente: Autoría propia (2021) 


\section{Discusión}

\subsection{Un paisaje fluvial degradado}

La persistencia anual del incumplimiento sobre la calidad del agua registra incidencias sobre los límites de DQO y DBO, aunque desde el 2018 no hay registros de contaminado o muy contaminado. Sin embargo, los efectos a la salud humana son persistentes. Por otra parte, la expansión de la zona urbana sigue en aumento, pese a no resolver el problema de desocupación de vivienda (Figura 8) dadas las condiciones de los nuevos asentamientos.

El constante impacto con el que viven los centros urbanos del entorno del río supone un estado de alarma extraordinario. Un problema de tal envergadura debería ser atajado desde varios ángulos y con diversas medidas; la implementación de las medidas de recuperación debería considerar un entendimiento completo, de lo contrario pueden caer en una perspectiva sesgada, como en el caso del río Medellín, Colombia, el cual fue acogido por un mercado político estratégico en el que la autoridad, ambiental en su caso, implementa objetivos políticos en miras de mantener, o bien obtener, prestigio ante la ciudadanía (García, 2010), y en donde, además de ganar legitimidad púbica, se afianza con confianza con las instituciones de conveniencia, puesto que las estrategias no se configuran para resolver el problema de raíz ni de forma tajante. La importancia de no perder e foco de atención a la gente de las poblaciones dañadas, así como de los impactos ambientales a la zona, debiera ser el corazón del que emerjan propuestas racionales, humanas y coherentes a la magnitud del problema.

En la situación del río Santiago es importante visualizar los patentes efectos sobre la población, entendiendo que cuando se genera un crecimiento urbano hacia zonas degradadas o contaminadas, se fomenta la marginación y colaterales efectos como el abandono de la vivienda. La escala del problema podría llevar a plantear un seguimiento legislativo sectorizado y descentralizado mediante normativa local que controle la cantidad y toxicidad de los vertidos; esfuerzos conjuntos y colectivos por parte de la industria que puedan mejorar la urgente situación sanitaria; alternativas de gestión que sirvan para revertir la situación de determinados escenarios en los que puedan verse beneficiados no solo el paisaje natural y la salud urbana, sino erigirse como una herramienta de valor económico y político.

\subsection{De vertedero a río}

A la sobreposición de las diferentes capas que deberían constituir la solución al problema, debería sumarse aquellas de tipo urbano-territoriales que tomen en consideración la potencialidad del río Santiago como un elemento fundamental en los ciclos ecosistémicos, agua, sedimentos, así como incidir en la importancia de su papel en los ciclos biogeoquímicos. El paisaje fluvia debería ser puesto en revalorización como un elemento arraigado a la historia y cultura de las regiones que, por tanto, necesitan una atención prioritaria.
Los cruces de información que se deben compaginar en este caso tan complejo deberían incluir un cambio de normativa de descargas en el país, sectorizada por giro industrial, ya que este es el argumento que impide homologar un parámetro, así como también modificaciones en las lecturas de calidad de agua, que incluyen vigilancia e inspección (McCulligh, 2017). Las modificaciones a la NOM-001-SEMARNAT-1996 son imprescindibles para garantizar que la calidad de agua tiene las condiciones de salubridad, pues es inconcebible que una normativa con 25 años de vigencia siga permaneciendo como el máximo regulatorio ante una situación tan grave y con consecuencias altamente cuantificables en el estado, y que además se extiende a problemáticas en los estados adyacentes.

La transformación que posibilitaría la recuperación de Río Santiago podría establecerse en los siguientes tres órdenes:

1) Recuperación de cadenas tróficas: En dónde a través de la disminución de los agentes contaminantes antes mencionados se pueda recuperar el equilibrio de oxígeno, nitrógeno y regulación del $\mathrm{pH}$, y con esto una recuperación paulatina de las comunidades ícticas. De forma paralela, la recuperación de la flora en la orilla del río beneficiaría la fauna silvestre, que se encuentra mayormente desaparecida; la incorporación de parámetros naturales de control podría ser para el río Lerma como para otros casos en México una forma de implementar sistemas biológicos de monitoreo como aprendizajes que se obtienen de aquellos casos de control de plagas biológicas. Entender la naturaleza como un agente sabio que reconoce la vida ante los entornos en los que se propician es un acercamiento amable a los espacios naturales.

2) Aprovechamiento productivo del recurso hídrico: $\mathrm{E}$ cierre de la cuenca Lerma-Chapala se debió a la alta demanda. Si bien los requerimientos son altos por parte de la agricultura, los cuales representa alrededor del $80 \%$ en la zona (Vargas-Velázquez, 2010), no fue sino debido a otras dificultades asociadas a la falta de regulación y la mala calidad de agua, ya no solo del río sino de los correspondientes mantos freáticos, de la zona productiva Santiago Ixcuintla ubicada en Nayarit. "De acuerdo con estos resultados, la zona norte (municipios de Acaponeta, Rosamorada, Ruiz, San Blas, Santiago Ixcuintla, Tecuala y Tuxpan) es la que mayor número de casos de intoxicaciones registró en 2007" (González-Arias et al., 2010, p.225), cuestión directamente asociada al uso de plaguicidas. Por tanto, se estima que, de posibilitar reaperturas del cauce fluvial con las correspondientes restricciones de uso, sin superar el ciclo hidrológico de la cuenca, así como el monitoreo a los núcleos agrarios en relación con el uso de herbicidas, insecticidas y fungicidas, se facilitarían nuevas posturas de aprovechamiento del recurso para estas zonas productivas. Esto con la finalidad a largo plazo de transformar los intereses de uso de suelo en las cercanías del río en complemento y soporte a la línea 1) Recuperación de cadenas tróficas. Es interesante poder asociar las intenciones económicas de la zona a los intereses ecosistémicos del río, pues no existe nada más complejo que la coexistencia de la industria con un sistema tan frágil y vulnerable como lo es el paisaje fluvial. Por tanto, junto al aprovechamiento 
productivo del recurso hídrico, debería sumarse la idea de reconvertir la utilización del suelo con fines de producción únicamente primaria, y esto, claro, de la mano con el diálogo al sector industrial, entendiendo que la afectación a este sector tiene una dimensión difícilmente equiparable a la afectación social causada.

3) Mejora de las condiciones socio-urbanas: La salud humana y la integridad ambiental deberían ser los principales motores que transformen las relaciones naturales y urbanas. Un entorno saludable debe generar un círculo virtuoso de relaciones y multiplicar las posibilidades de regeneración ambiental. En el caso de las comunidades adjuntas al Río Santiago, deben poder regresar al disfrute del ambiente natural con la tranquilidad de un entorno en condiciones que permita la vida, y a su vez contribuir en la permanencia de las características naturales; coexistencia de dos hábitats en donde la simbiosis y la resiliencia permitan el desarrollo de ambas. Este aspecto, aunque no está dirigido hacia recomendaciones puntuales, sí es un punto base fundamental hacia el entendimiento del disfrute de la zona por parte de las comunidades, manifestándose en la salud humana y las garantías asociadas a una vida digna.

Un planteamiento integral que pueda interrelacionar las condiciones sociales, los derechos humanos, las necesidades urbanas y los factores ambientales, puede propiciar respuestas que reúnan una visión más competente; posibilitar un nuevo aproximamiento metodológico en el que se conciban integraciones más racionales de las personas con su entorno, como una analogía en donde el encuentro de dos ecosistemas generan una tercera zona de encuentro, conocida como ecotono. Podría pensarse en una reinvención de las zonas de encuentro entre los ecosistemas, producto de artificio en un diálogo fundamentado por el respeto, y en un marco regulatorio que garantice la sanidad ambiental y humana con su relación a los ecosistemas naturales, siendo latente la generación de una zona de encuentro que intensifique las bondades de los ecosistemas hacia un nuevo planteamiento lingüístico y a una hipótesis teórica del urbatono. Este concepto surge con respecto a las relaciones de ecosistemas naturales, y es también extensible a la diversidad de confines con bilateralidad de usos, intereses y actuaciones en los que el artífice, en la totalidad de los casos, termina por alterar de forma cas irreversible la realidad del biotopo existente. Los diversos argumentos actuales sobre interfaces, capas o relaciones entre la ciudad y un río, nos trasladan la duda sobre la posibilidad de hibridar ecosistemas, específicamente entre el natural y el artificial, aunque se entienden como campos físicos con fuerzas diversas y contradictorias, dado que muchos límites entre la naturaleza y las urbes han sido resueltos de forma tosca tras ejercicios fallidos. No obstante, deberían ser objeto de revisión para vislumbrar nuevos sistemas de relaciones que permitieran una transición entre los dos hábitats más eficaces y con respeto por la vida.

El contexto actual refleja cada vez más territorios naturales donde incluso los más conservados son sometidos a la lógica urbana dominante, ríos utilizados como vialidades o ríos como espacios residuales, siendo entendidos expresiones antropocéntricas y no como relaciones saludables. Como señalan López-Mares et al.: "la producción social de la naturaleza, vista desde un enfoque del ciclo hidro-social, no solo implica la domesticación de lo natural para fines sociales, sino también de la reconfiguración social desde lo natural en una relación dialéctica" (2019, p.45). Aunque ya se investiga sobre las potencialidades de las ciudades como escenario físico o biotopo, como dadores de hábitat para las especies no humanas (Real-Giménez, 2009), esto es visible a día de hoy por una minúscula minoría de casos en ciudades, y mayormente desde la perspectiva teórica. Si bien la teoría a la aplicación dista, sería interesante tomar en consideración posibles escenarios de recuperación urbana mediante nuevas directrices de interacciones con el entorno como parte de robustas estrategias de mitigación y recuperación de condiciones saludables del territorio.

\subsection{Industria como motor de cambio}

El incómodo diálogo entre la importancia económica de la zona industrial a la orilla del río Santiago y los severos impactos que genera a las condiciones ambientales, lo convierte en una paradoja sobre la existencia de la industria en la zona. Este debate establece una lucha insostenible para empresarios y agricultores. Sin embargo, desde el planeamiento del territorio, pueden diagnosticarse algunas áreas de oportunidad que generen una conciliación entre ambos sectores productivos y las necesidades ciudadanas. Si bien, el estado actual de los vertidos residuales es un problema del orden sistémico sobre la legislación y gestión de la normativa, es importante considerar que, si la estrategia de la zona fomenta destacar el papel industrial como herramienta de fortalecimiento social, podría resultar un planteamiento reivindicativo para la producción de un entorno urbano que se enriquezca por la presencia industrial.

Existen algunos buenos ejemplos sobre la recuperación de la sanidad ambiental del paisaje fluvial a través de la reconversión industrial. Por ejemplo, la Ría del Nervión en Bilbao es un profundo ejemplo de la gestión ambiental y territorial a la que diversos grupos de presión se oponían. O el caso del río Loira, en Nantes, cuya revitalización del territorio industrial estuvo marcada por la preservación del patrimonio industrial, pero a su vez ratificando el futuro para la ciudad industrial.

Las políticas urbanas y de vivienda deben ser capaces de generar una interacción positiva entre las zonas industriales y las residenciales, con el objetivo de favorecer el fortalecimiento de las comunidades y de contribuir a una planeación urbana integral. Asimismo, no hay que olvidar las particularidades actuales de las poblaciones del río Santiago, poblaciones marginadas y por tanto sensibles a las modificaciones urbanas. Por ello, el trabajo urbano con las responsabilidades de la administración pública que corresponden de la mano con la participación ciudadana debe significar una plataforma segura para escuchar las necesidades y el sentir de los pobladores.

El camino hacia la recuperación del río Santiago y de sus alrededores debe considerarse una labor constante que permita de forma progresiva la mejora y mitigación de los múltiples daños acumulados. 


\section{Conclusiones}

Es importante no dejar de lado la dinámica del paisaje fluvial, y menos en su interacción urbana, pues constituye un potente recurso de alto contenido educativo, y de aprendizaje sensible hacia las transformaciones simbióticas de la ciudad con el entorno. Se debe revalorizar la constitución de los ríos con respecto a la gestión urbana y económica de las ciudades, buscando establecer un diálogo basado en el cohabitar y en el respeto a los límites ecológicos y de la fragilidad ecosistémica. La solución para el río Santiago no yace en pequeñas acciones paliativas, sino que debe considerar potentes acciones de infraestructura de saneamiento y de rigor normativo. Las decisiones territoriales deben partir desde la reflexión del futuro de las comunidades y considerar estrategias urbanas que permitan el uso de los servicios ecosistémicos de una forma racional para la recuperación ecosistémica.

\section{Recomendaciones}

Desde el análisis urbano podría realizarse otro tipo de acercamientos en una escala menor, para realizar estudios sectorizados que permitan la comprensión de las condiciones y de las particularidades de las diversas comunidades. De los mapas urbanos estudiados en el presente documento podrían desprenderse investigaciones futuras bajo la misma metodología, a manera de seguimiento al crecimiento poblacional y sus efectos en las comunidades próximas al río. Asimismo, se observan potencialidades de investigación para el entendimiento de la variación de los resultados obtenidos sobre la calidad del agua a lo largo del río Santiago hacia su desembocadura en el Océano Pacífico, pudiendo estar asociados a factores de disolución, permeabilidad, o de afinidad química de los vertidos en él con la morfología y tipología de suelo del río.

Cómo citar este artículo/How to cite this article: Vigil de Insausti, A., Herrera Piñuelas, I. y Esteller Agustí, A. (2021). Río Santiago, un paisaje fluvial en colapso como oportunidad de recuperación urbana. Estoa. Revista de la Facultad de Arquitectura y Urbanismo de la Universidad de Cuenca, 10(20), 17-29. doi: https:// 10.18537/est.v010.n020.a02

\section{Referencias bibliográficas}

Comisión Nacional del Agua (2012). Atlas del agua en México 2012. Subdirección General de Programación. Comisión Nacional del Agua.http://www.conagua.gob. $\mathrm{mx} /$ CONAGUA07/Publicaciones/Publicaciones/SGP-3612.pdf

Cotler, H., Hiriart M. y Sánchez, J. (Eds.), (2006). Atlas de la cuenca Lerma-Chapala. Construyendo una visión Conjunta. Secretaría de Medio Ambiente y Recursos Naturales-Instituto Nacional de Ecología-Universidad Nacional Autónoma de México-Instituto Nacional de Ecología, México.

de Anda, J. y Maniak, U. (2007). Modificaciones en el régimen hidrológico y sus efectos en la acumulación de fósforo y fosfatos en el lago de Chapala, México. Interciencia, 32(2), 100-107.

Domínguez Cortinas, G. (Ed.) (2010). Propuesta metodológica para la implantación de una batería de indicadores de salud que favorezcan el establecimiento de programas de diagnóstico, intervención y vigilancia epidemiológica en las poblaciones ubicadas en la zona de influencia del proyecto de la presa Arcediano en el estado de Jalisco. Universidad Autónoma de San Luis Potosí, Comisión Estatal de Agua de Jalisco. https:// transparencia.info.jalisco.gob.mx/sites/default/files/ INFORME\%20FINAL\%20ARCEDIANO_CEA_UEAS JALISCO_2011_1\%20-\%20copia_opt.pdf

EFE (3 de marzo de 2020). El río Santiago, un caudal tóxico que ha envenenado a cientos en México. Agencia EFE. https://www.efe.com/efe/usa/mexico/el-rio-santiagoun-caudal-toxico-que-ha-envenenado-a-cientos-enmexico/50000100-4187233

El Informador (13 de febrero de 2020). Mil 430 muertes por contaminación en El Salto en 12 años: colectivo. El Informador. https://www.informador.mx/jalisco/Mil-430muertes-por-contaminacion-en-El-Salto-en-12-anoscolectivo-20200213-0121.html

García Cardona, A. (2010). La contaminación del río Medellín (2006-2011): un mercado político. Lecturas de Economía, (72), 169-194. https://revistas.udea.edu.co/ index.php/lecturasdeeconomia/article/view/6520

González-Arias, C. A., Robledo-Marenco, M. de L. Medina-Díaz I. M., Velázquez-Fernández, J. B., GirónPérez, M. I., Quintanilla-Vega, B., Ostrosky-Wegman, P., Pérez-Herrera, N. E. y Rojas-García, A. E. (2010). Patrón de uso y venta de plaguicidas en Nayarit, México. Revista Internacional de Contaminación Ambiental, 26 (3), 221 228. https://www.revistascca.unam. mx/rica/index.php/ rica/article/view/20459

Guevara, S., Arellano, O. y Fricke, J. (2014). Ríos tóxicos: Lerma y Atoyac. La historia de la negligencia continúa. Greenpeace. https://agua.org.mx/wp-content/ uploads/2018/02/Rios-toxicos-Lerma-y-Atoyac Greenpeace.pdf 
Guzmán-Arroyo, M. y Peniche-Camps, S. (2009). Estudios de la Cuenca del Río Santiago. Un enfoque multidisciplinario. Editorial Páramo. https://www.eumed. net/libros-gratis/2010f/859/859.pdf

Instituto Mexicano de Tecnología del Agua. (2009). Estrategia General para el rescate ambiental y sustentabilidad de la Cuenca Lerma-Chapala. Informe final. Instituto Mexicano de Tecnología del Agua SEMARNAT. https://biblioteca.semarnat.gob.mx/janium/ Documentos/Ciga/libros2009/EGRASCLCH.pdf

López-Hernández, M., Ramos-Espinoza, M. y CarranzaFraser, J. (2006). Análisis multimétrico para evaluar contaminación en el río Lerma y lago de Chapala, México, Hidrobiología, 17 (1), 17-30.

López-Mares, L.M., Lozano-de Poo, J.M., Torre-Silva, F., Rodríguez-Santiago, J. y López-Fraga J.A. (2019). E ciclo hidro-social de los ríos urbanos: Transformaciones al paisaje hídrico en San Luis Potosí, México. Revista de Ciencias Ambientales. Tropical Journal of Environmental Sciences. 53 (1), 45-69. http://dx.doi.org/10.15359/ rca.53-1.3

Martínez- González, P. y Hernández-González, E. (2009). La contaminación del Río Santiago. Impactos en el bienestar de los habitantes del Municipio de El Salto, Jalisco. XXVII Congreso de la Asociación Latinoamericana de Sociología. VIII Jornadas de Sociología de la Universidad de Buenos Aires (pp. 709-729). Asociación Latinoamericana de Sociología.

McCulligh, C. (2013). La no regulación ambiental contaminación industrial del río Santiago en Jalisco. Observatorio del desarrollo, 2 (7), 22-29. https://doi. org/10.35533/od.0207.cm

McCulligh, C. (2017). Alcantarilla del progreso: Industria y estado en la contaminación del Río Santiago en Jalisco [Tesis de doctorado, Universidad Autónoma de Zacatecas].

McCulligh, C., Páez, J. y Moya, G. (2007). Mártires del Río Santiago. Instituto Mexicano para el Desarrollo Comunitario.

Quiroz-Castelán, H., Mora-Zúñiga, L., Molina-Astudillo, I. y García-Rodríguez, J. (2004). Variación de los organismos fitoplanctónicos y la calidad del agua en el Lago de Chapala, Jalisco, México. Acta Universitaria 14(1), 47-58.

Real-Giménez, R. (5 de noviembre de 2009). La ciudad y la biodiversidad urbana [Conferencia]. OMAU Observatorio de Medio Ambiente Urbano, Málaga, España.

Vargas-Velázquez, S. (2010). Aspectos socioeconómicos de la agricultura de riego en la cuenca Lerma-Chapala, Economía, Sociedad y Territorio, X (32), 231-263.

https://agua.org.mx/wpcontent/uploads/2014/04/

AspectosSocioeconomicosAgriculturaLermaChapala.pdf 